\title{
Circuit-mode Features of the Distribution Network in the SAIDI and SAIFI Forecast
}

\author{
Yuri Kakosha ${ }^{1}$, Lyudmila Myshkina ${ }^{1, *}$, and Inna Sabadash $^{1}$ \\ ${ }^{1}$ Novosibirsk state technical university, Karla Marksa, 20, Novosibirsk, Russia
}

\begin{abstract}
The adoption of any technical solutions at the design stage of the distribution network significantly affects the change in the indicators of uninterrupted power supply to consumers: SAIDI and SAIFI. However, methodological support for their determination for the future is not enough. The development of an appropriate methodology and its implementation into design practice is necessary, this is especially important in connection with the appearance of distributed generation, which has a significant impact on the circuit-mode conditions of power supply systems. The developed methodology makes it possible to determine the most effective from the many possible ways of integrating distributed generation and local power supply systems, considering the of the technical condition indices, the structural and functional reliability of the distribution network and the existing mode restrictions, which is a necessary element of development management during design.
\end{abstract}

\section{Introduction}

Electric networks diagrams shall ensure the necessary reliability of power supply, transfer of the required amount of electricity of the required quality to the consumers, possibility of the further network development and connection of new consumers, convenience and safety of operation [1]. Therefore, requirements to indicators demonstrating reliable power supply to the consumers are of primary importance for the distribution electric networks.

Indicative reliability indexes have been used at the operational stage since 2012. These include SAIDI (System Average Interruption Duration Index) and SAIFI (System Average Interruption Frequency Index) indexes demonstrating the average duration and frequency of interruptions in power supply to consumers in the area of electric networks, values of which affect the electricity transmission service tariffs [2].

Currently, when designing distribution networks, a technical and economic comparison of power supply options is used, which is based on the of the consumer reliability category concept. However, to a great extent this situation does not correspond to the current state of the distributed generation, electric network digitalization and changes in the control system. It is required to introduce additional criteria to increase technical solutions validity at the stage of managing the distribution networks development. It is proposed to introduce SAIDI and SAIFI forecasted reliability indexes, which values, as noted above, affect economic efficiency of the company.

The need to combine centralized and decentralized control presupposes creation of automation that influences the circuit and regime operating conditions of the distribution network in addition to conventional regime and emergency control devices. This requires to improve the distribution network design process in order to form certain properties - reliability, recoverability, controllability, durability and others. Consequently, tasks of developing the appropriate methodological support, regulatory framework, new means and ways to achieve the set goal are relevant tasks, which determines relevance of the research carried out by the authors, as well as scientific and practical significance of the obtained results.

The purpose of the work is to develop a methodology for calculating the forecasted values of uninterrupted power supply indexes (SAIDI and SAIFI) taking into account the circuit and regime changes in $10 \mathrm{kV}$ distribution network section (DSS) when designing:

- new network equipment;

- connection to the distributed generation network.

DSS is an element of the power supply system for consumers powered from one power supply center. Obviously, selection of the circuit and power lines composition, number of distribution point stations, number and capacity of $10 / 0.4 \mathrm{kV}$ transformer points and switching equipment depends on two factors:

- capacity of power transformers of the district $110 / 10 \mathrm{kV}$ substation and $35 / 10 \mathrm{kV}$ substation;

- number and capacities of consumers, their distribution over the territory;

- distribution of consumers by the power supply reliability category.

Scientific novelty consists in taking into account technical condition of network equipment when

\footnotetext{
* Corresponding author: Lsmyshkina@gmail.com
} 
calculating uninterrupted power supply indexes, as well as in developing a method for determining "critical" hubs and requirements to justify network redundancy or connection to the distributed generation network.

Practical significance consists in supplementing the methodological base for the development of consumers technological connection diagrams and power distribution schemes by power plants. It is noteworthy that obtained results make it possible to solve a set of tasks for the creation of local energy systems and their integration with the UES for synchronous parallel operation with the possibility of separating them to maintain uninterrupted power supply in case of $35 \mathrm{kV}$ and higher network technological failure.

\section{Status of the challenge addressing}

To assess power equipment reliability and service continuity $35 \mathrm{kV}$ and higher network, which form the federal and regional power supply systems, the following methods and guidelines are currently used:

- Methodology for assessing technical condition of the main technological equipment and power transmission lines of power plants and electric networks approved by Order No. 676 of the Ministry of Energy of Russian Federation dated 26.07. 2017 (as amended on 17.03. 2020) [3];

- Methodological guidelines for calculating the probability of failure of a functional unit and a piece of the main technological equipment and assessing consequences of such a failure approved by Order No. 123 of the Ministry of Energy of Russian Federation dated 19.02.2019 [4].

Both methodologies cover the following technological equipment of electricity generation facilities, which technical condition is being assessed:

Steam turbines with the installed capacity of $5 \mathrm{MW}$ and higher; Steam (power-plant) boilers providing steam to steam turbines with the installed capacity of $5 \mathrm{MW}$ and higher; Hydraulic turbines with the installed capacity of $5 \mathrm{MW}$ and higher; Gas turbines with the installed capacity of $5 \mathrm{MW}$ and higher; Hydrogenerators with the rated power of $5 \mathrm{MW}$ and higher; Turbine generators with the rated power of $5 \mathrm{MW}$ and higher; Power transformers with voltage of $110 \mathrm{kV}$ and higher; Power transmission lines (hereinafter, transmission lines) with the voltage of $35 \mathrm{kV}$ and higher (hereinafter, main technological equipment).

The available methodology [3] determines the procedure for assessing technical condition of the main technological equipment and determining optimal type, composition and cost of technical impact on the equipment. One of the main purposes of the technical condition index (TCI) determining methodology is to address challenges of decision-making on the technical impact on technological equipment, i.e. the methodology is applied at an operating facility taking into account statistical data collected during the operation. The available Methodological guidelines [4] determine the procedure for calculating the probability of failure of a functional unit and a piece of the main technological equipment of power plants and electric networks, as well as the procedure for assessing consequences of such a failure. The methodology ensured accelerated determining of uninterrupted power supply indicative indexes.

The calculation of the probability of failure of a piece of the main technological equipment and (or) its functional unit shall be based on:

- Forecasting changes of technical condition index of the piece of the main technological equipment

- Forecasting probability of failure of functional units of the piece of main technological equipment

- Forecasting probability of failure of the piece of main technological equipment.

The mentioned methodologies [3] and [4] are applied to $35 \mathrm{kV}$ and higher electrical networks (power supply centers) characterized with high observability and sufficient scope of statistical documents for the main technological equipment. Circuit and regime peculiarities and structure of these networks allows determining technical condition indexes for each piece of equipment, as well as taking into account the forecasted reliability indexes when designing. However, there is a number of differences between $10 \mathrm{kV}$ distribution networks and 35-220 kV supply networks, which makes it impossible to apply methods [3] and [4] in these networks. Let's analyse these differences. One of the main features of power distribution networks for domestic and agricultural consumers is their ramification and length. This is due to the variety of connected consumers (both in size and composition of loads), proximity of the connection points, mainly radial method of loads connection and the lack of these networks future development schemes. Structure of the distribution networks is ramified and complex due to many switching devices and overhead lines, which leads to frequent technological failures of these networks. As a result, failures in $6-10 \mathrm{kV}$ networks cause about $70 \%$ of all power supply disturbances to consumers, which reduces technical efficiency of these networks [5].

As an example, we can take a part of the real power supply scheme of Sverdlovsk Region of Irkutsk (REN) of the Southern Electric Networks of JSC "IESK" (see Fig.1). Based on the circuit analysis results, one $35 / 6 \mathrm{kV}$ power center supplies 44 transformer substations of $6 / 0.4 \mathrm{kV}$, two Central Distribution Point Stations of $6 \mathrm{kV}$ and more than $60 \mathrm{~km}$ of $6 \mathrm{kV}$ lines. At the same time, maximum power of the load connected to the power supply points of $6 / 0.4 \mathrm{kV}$ transformer substation is 9 MW, although 2x10 MW transformers are installed in the power supply centers. According to the current rules, size of the connected load is limited in terms of reliability requirements, i.e. based on $n-1$ criterion. A large number of main electrical equipment with different technical characteristics makes it impossible to apply the available methods for calculating the TCI and the probability of the equipment failure. Calculation of distribution networks reliability indexes is also complicated by the lack of periodical examination of the 
main equipment necessary parameters, and as a result, impossibility of annually calculation of changing TCI for each piece of the equipment.

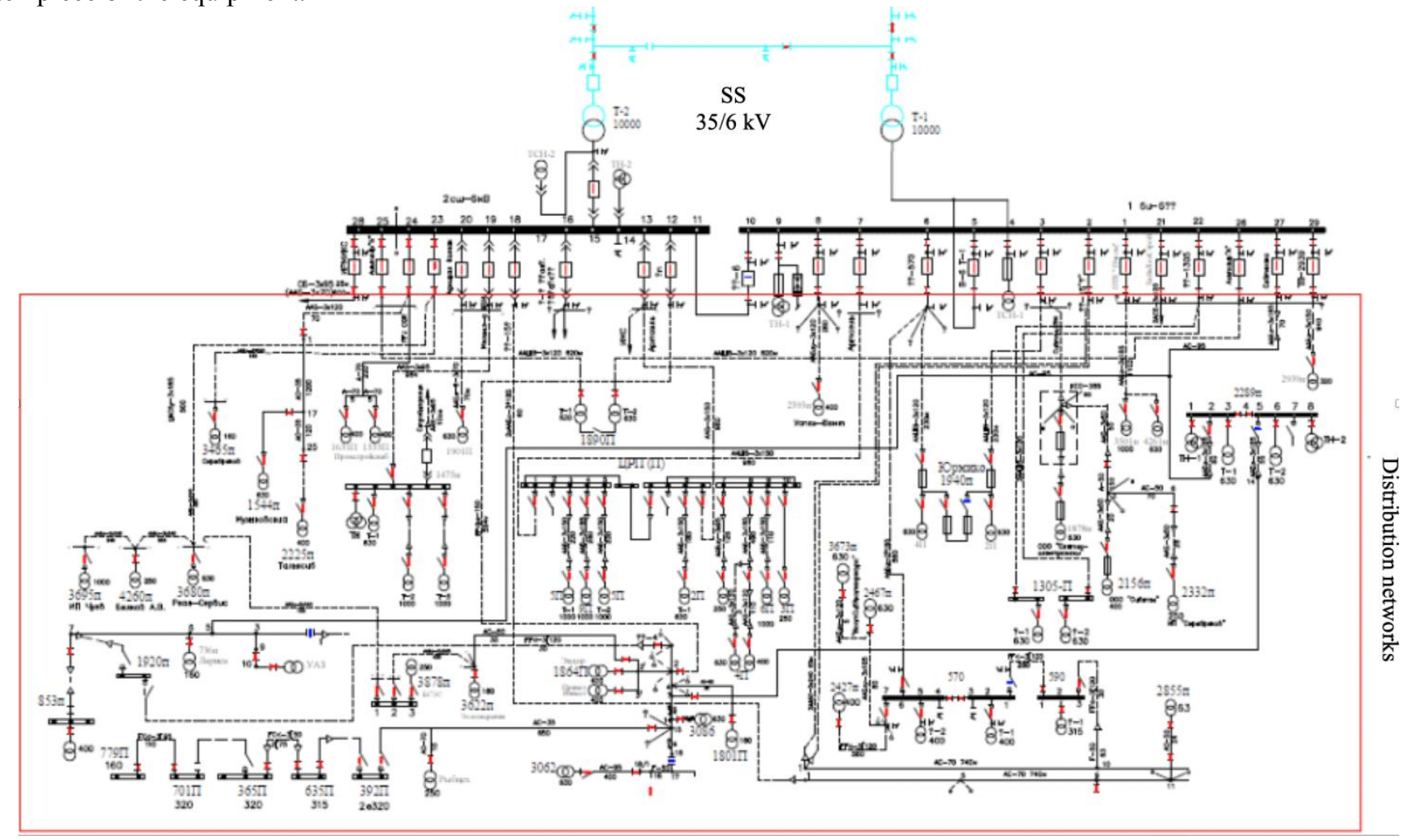

Fig. 1. Part of REN power supply diagram

Uninterrupted power supply depends mainly on distribution networks, and taking into account the forecasted reliability indexes of these networks can increase validity of decision-making at the design stage. However, methods applied today prevent from the task fulfilling in distribution networks, i.e. to determine the forecasted SAIDI and SAIFI indicative reliability indexes at the stage of distribution network development management. Consequently, it is required to develop additions that will allow using the methodologies for calculating technical condition indexes and probability of failure-free operation of electrical equipment in the distribution network, as well as determining promising indicative indexes of uninterrupted power supply.

\section{Main provisions of the proposed methodology}

First of all, when designing electric distribution networks, conditions of reliable and high-quality power supply to consumers must be observed, in contrast to the main system-forming networks, where compliance with technical conditions ensuring safety and reliability of electrical power systems and maintaining stable synchronous operation in normal and post-emergency regimes is of primary importance.

In addition to the existing methods for determining forecasted indicative power supply reliability indexes in distribution networks, it is proposed to calculate generalized TCI for groups of equipment (elements) of the distribution network rather than for each piece of equipment individually.

\subsection{Generalized index of the distribution network technical condition}

Main elements of $10 \mathrm{kV}$ distribution network are a power transformer, cable line, an oil circuit breaker. Table 1 shows main functional units of the elements identified in the frame of this work.

Table 1. Functional units of distribution network elements.

\begin{tabular}{|c|c|}
\hline Facility & Functional units \\
\hline $\begin{array}{c}\text { Power } \\
\text { transformer }\end{array}$ & $\begin{array}{c}\text { Insulation system; transformer windings; } \\
\text { magnetic circuit; high-voltage bushing; } \\
\text { voltage regulation system }\end{array}$ \\
\hline Cable line & $\begin{array}{c}\text { Auxiliary equipment; terminal and connecting } \\
\text { couplings, power cable }\end{array}$ \\
\hline $\begin{array}{c}\text { Circuit } \\
\text { breaker }\end{array}$ & $\begin{array}{c}\text { Drive, arc-suppression chamber, contact } \\
\text { system }\end{array}$ \\
\hline
\end{tabular}

The adopted methodology for assessing technical condition of the main technological equipment [3] does not indicate how certain weighing factors were assigned for the functional units taken into account for the functional units technical condition index calculation.

The proposed method envisaged weighting coefficients of the network element functional units to be 
determined based on the expert assessments and statistical data of technological disturbances caused by the failure of functional units corresponding to this element.

The final score was determined by the method of expert assessments and the Saati method [6] with the introduction of coefficients demonstrating the experts competence. Expert assessments shall be drawn up for each distribution network section taking into account its peculiarities. Authors of the study assumed that the boundary values of the scoring scale characterizing functional units technical condition and level of performance of the required functions. functional units scoring multiplied by the functional units weight allows obtaining the generalized TCI. Value of the generalized TCI will be applied in determining the prospective values of the indicative power supply reliability indexes.

\subsection{Generalized index of the distribution network technical condition}

When calculating forecasted probability of failure of the main technological equipment functional unit or a PTL segment for a forecasted period of 5 years, methodology [4] uses a correction factor that takes into account the ratio of the value of the main technological equipment functional unit technical condition index or the PTL segment before and after the last technical impact or during the previous and current calendar year.

However, system of collecting information on the parameters characterizing technical condition of the equipment currently used in distribution networks does not allow obtaining reliable data. It has been established that data collection process is not conducted systematically, in a timely manner, and does not provide formal data analysis. Causes of numerous network elements failures (up to 50\%) have not been identified. This indicates an unsatisfactory condition of the system for collecting repair and operational information at the power grid complex facilities, as well as insufficient number of technical diagnostics and automated monitoring systems [7]. All of the above indicates impossibility of obtaining a reliable correction factor value that demonstrates impossibility to determine network functional unit failure probability.

In this regard, the proposed methodology provides for the correction of $\lambda_{i}$ distribution network element failure rate determination, which is proposed to be used taking into account the required and actual values of the technical condition generalized index:

$$
\lambda=\lambda_{s}\left(\frac{T C I_{g}{ }^{r}}{T C I_{g}}\right)
$$

where $\lambda s$ is the statistical value of the power supply system element failure rate; $T C I_{g}{ }^{r}$ - the required value of the technical condition generalized index, $T C I_{g}{ }^{r}$ is taken equal to 85 points for the distribution network elements since it corresponds to the running-in period; $\mathrm{TCI}_{g}{ }^{c}$ - distribution network element technical condition generalized index under actual operating conditions.
Therefore, to calculate idle time of the power supply system element $(\mathrm{T})$, the well-known expression shall be taken:

$$
T=\lambda t_{r}
$$

where $t_{r}$ - is the recovery time of the $\mathrm{i}$-th element of the power supply system.

Forecasted System Average Interruption Duration Index $\left(S A I D I_{F}\right)$ for the local grid company supplying energy to the point of delivery, taking into account the distribution network, shall be determined by the formula:

$$
S A I D I_{F}=S A I D I_{C}-\left[\left(\frac{\sum T_{i} \cdot N_{i}}{N_{\max }}\right)^{C}-\left(\frac{\sum T_{i} \cdot N_{i}}{N_{\max }}\right)^{F}\right]
$$

Forecasted System Average Interruption Frequency Index $\left(S A I F I_{F}\right)$ for the local grid company supplying energy to the point of delivery, taking into account the distribution network, shall be determined by the formula:

$$
S A I F I_{F}=S A I F I_{C}-\left[\left(\frac{\sum \lambda_{i} \cdot N_{i}}{N_{\max }}\right)^{C}-\left(\frac{\sum \lambda_{i} \cdot N_{i}}{N_{\max }}\right)^{F}\right]
$$

where $\mathrm{C}$ - is the indexation of the current power supply system condition; $F$ - indexation of the forecasted predicted supply system condition; $\lambda_{i}$ - failure rate of the i-th critical element of the power supply system, h; $N_{i}$ the number of delivery points that disconnected as a result of a technological upset due to the failure of the ith critical element of the power supply system, pcs; $N_{\text {max }}$ - number of delivery points in the power supply system, pcs; $T_{i}$ - idle time of the i-th critical element of the power supply system;

Power supply system critical elements according to the methodology is the element, which failure causes the largest number of disconnected points of connection. To determine power supply system critical elements, it is necessary to determine the number of units switched off in case of the network i-th element $\left(N_{i}\right.$ in (3), (4)) failure. It is proposed to simulate the element failure with control of the steady-state electrical regime and registration of the disconnected units with RastrWin3, ANARES, Eurostag, ETAP or others.

$$
N_{i}=\max \left(N_{i_{g}}\right)
$$

where $N_{i g}$ is the number of disconnected consumer connection points in case of the g-th element of the $\mathrm{j}$-th type failure; $j=1 \ldots \mathrm{J}$ is the sequential number of the network element type (transformers, cable power lines, switches and other key type elements of the power supply system); $g=1 \ldots \mathrm{G}$ is the sequential number of the same type element, $G$ is the number of the same type elements. In this case, it shall be assumed that there are no interrelated failures, only single equipment failures shall be taken into account.

It can be noted that there are quite ambitious plans to reduce uninterrupted power supply indexes in Russia. The average SAIDI in Siberia as a whole by the end of 2019 amounted to 2.53 hours, as a result of Digital Transformation 2030 program implementation, this figure will decrease in 2024 to 2.39 hours, and in 2030 it will reach 2.22 hours, which is $1.1 \%$ per year. The average SAIFI in Siberia amounts to 1.63 units, owing to the program implementation, in 2024 this figure should decrease to 1.55 and in 2030 it should reach 1.44 [8], which is $0.9 \%$ per year. 
Obviously, the main influence on these indicators changing will be provided by technical solutions at the level of $0.4-10 \mathrm{kV}$ distribution networks, which emphasizes relevance of the proposed methodology and expediency of its practical application.

\subsection{Application of distribution network development management methodology}

The proposed methodology provides for calculation of the forecasted uninterrupted consumer power supply indexes, as well as makes it possible to take specific technical decisions aimed at increasing of distribution network reliability while managing its development.

Comparison of the forecasted continuity indexes allows identifying critical network elements, which failure causes disconnection of the greatest number of consumers (both in terms of power and in terms of power supply restoration), which allows taking appropriate measures to ensure redundancy of these network elements.

One of redundancy provision methods and the current trend in the development of networks [9] is the construction of distributed generation (DG). DG can be used to ensure reliability of the power system since its introduction decreases main and supply networks load, increases reserves the network transmission capability and exudes problem of local energy shortage in the areas of closed power centers [10]. DG introduction will help to significantly reduce investments in the distribution network development and increase power supply continuity.

The proposed methodology makes it possible to determine the most optimal generation connection points to improve reliability of power supply to consumers "under the threat" of power loss. Thus, it will increase reliability of the entire electric network. Also, it will introduce additional criteria for the DG capacity selection taking into account the need to ensure SS-1 redundancy not only of the part, but of the entire network. The methodology can also be used to determine optimal points of combining several local power supply systems into a single low-power system [11] and to create universal Microgrid, energy cells with an $\mathrm{AC}$ infrastructure [12].

Below is an example of the proposed methodology approbation by the authors, which makes it possible to judge the obtained results reliability.

\section{Example of the methodology approbation}

Approbation of the proposed methodology was carried out on the example of the residential microdistrict DSS, power supply of which is carried out from SS-1 (Fig. 2). The load structure is mainly domestic consumption with a small share of non-production enterprises related to the service sector. The total number of supply points in the analysed area is 108 , power of the connected load is $9.42 \mathrm{MW}$.

Based on the results of the structural and functional network reliability analysis using ETAP programming and computing suite, values of the continuity indexes of the analysed power supply system current condition were determined:

- $S A I D I_{C}=2.6420, \mathrm{~h}$;

- $\operatorname{SAIFI}_{C}=0.2411,1 /$ year

Further, having analysed steady-state regimes, critical elements, which failure causes maximum number of the disconnected delivery points, were determined:

- K-10, which causes disconnection of 12 delivery points,

- transformers TP-3438 causing disconnection of 9 delivery points;

- failures of circuit breaker do not cause customer interruption.

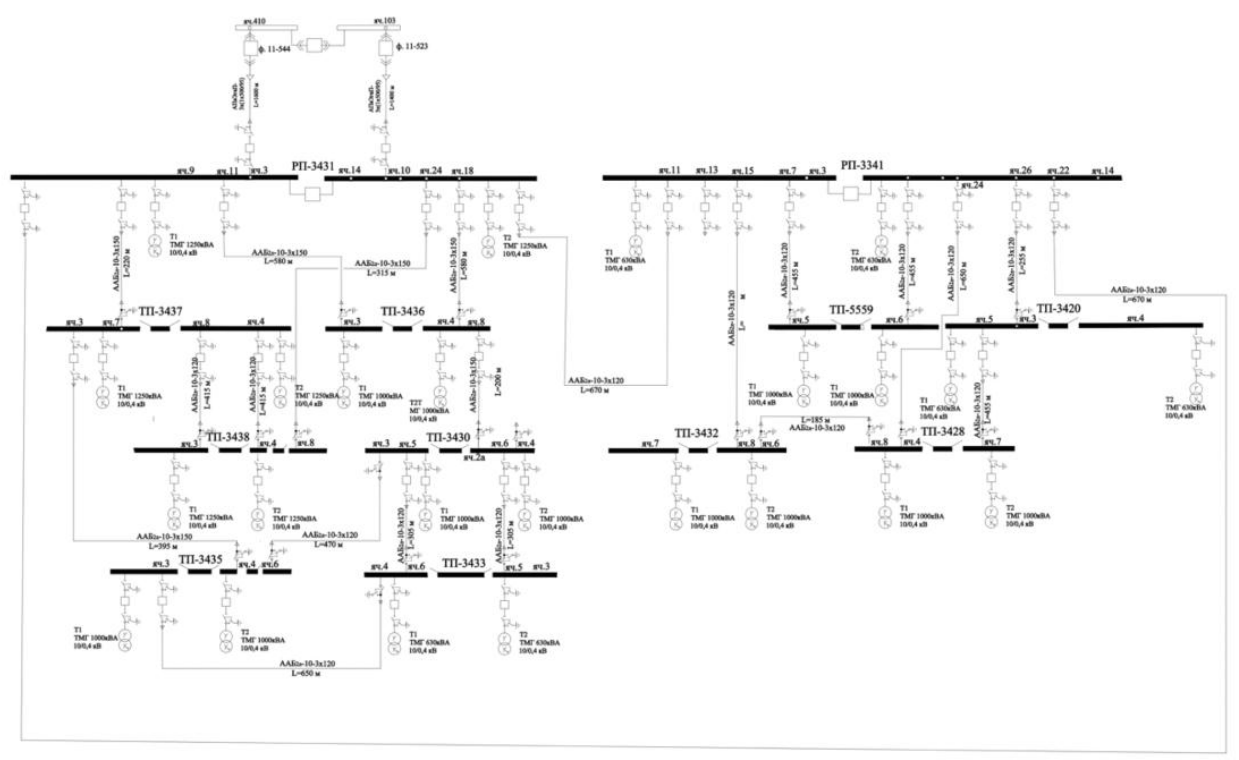

Fig. 2. Design single-line diagram of $10 \mathrm{kV}$ distribution network section 
Since the largest number of disconnected delivery points is caused by K-10 failure (the highlighted area in Fig. 3), a decision on the need to ensure the line redundancy was made.

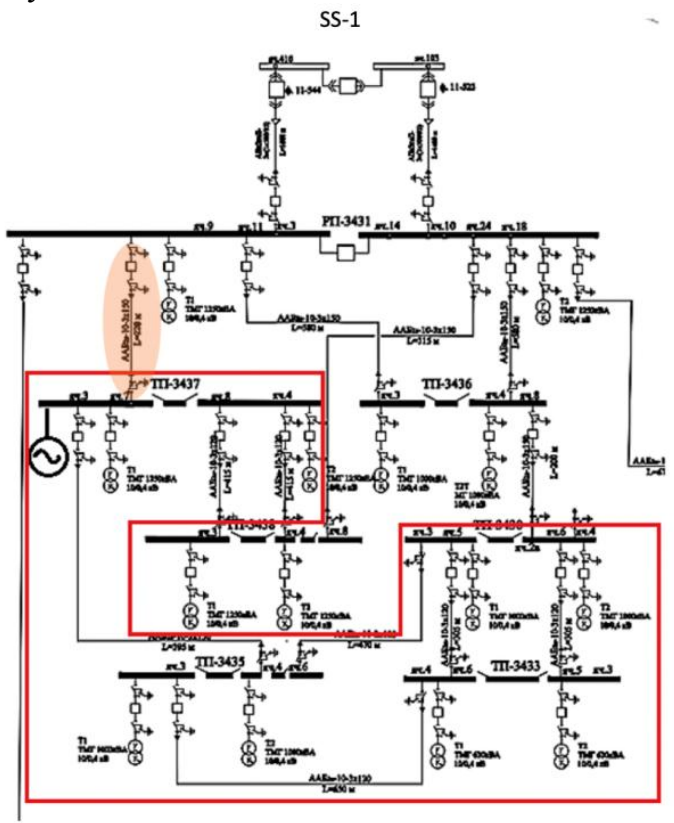

Fig. 3. Dedicated local power supply system.

DG connection and creation of the balanced local power supply system based thereon is considered as a measure to ensuring the improved uninterrupted power supply. Fig. 3 schematically demonstrated DG connection point (bus of TP-3437) with the highlighted local power supply system.

It is modelled that the DG performs network redundancy function, therefore, disconnecting K-10 does not lead to the load disconnection within the selected area, and then its power is determined based on the power of the load connected to the units considering $20 \%$ of the emergency reserve: $P_{D G}=4 \mathrm{MW}$.

Therefore, let's build a model providing for the connection of 4 Gas Engine Generators (GEG) of $1 \mathrm{MW}$ each. Performance indicators of the selected GEG: $t_{r}=$ $14.43 \mathrm{~h} ; \lambda=6.17$ times/year.

According to the proposed methodology, the forecasted uninterrupted power supply indexes were calculated when connecting the DG and creating a local power supply system (Table 2).

Thus, DG construction in the area of the analysed DSS allows improving SAIDI and SAIFI: the indicators decreased by $11.5 \%$ and $9 \%$, respectively. This proves effectiveness of the DG construction with the purpose to improve the uninterrupted power supply to consumers.

Table 2 demonstrates comparison of the results obtained using methodology proposed by the authors and results of calculations using ETAP programming and computing suite.

Deviations of the forecast indicators determined by the methodology did not exceed $5 \%$ of the indicators obtained using when calculating using ETAP programming and computing suite. This makes it possible to judge the methodology reliability and possibility of its application to increase validity of technical solutions decision taken at the stage of distribution networks development managing without use of additional expensive software.

Table 2. Comparison of the forecasted SAIFI and SAIDI values obtained by various methods.

\begin{tabular}{|c|c|c|c|}
\hline Method & Methodology & ETAP & Deviation, \% \\
\hline SAIDI & 2,3651 & 2,3632 & 0,1 \\
\hline SAIFI & 0,2211 & 0,2076 & 5,0 \\
\hline $\boldsymbol{\Delta}$ SAIDI, \% & 11,5 & 11,8 & - \\
\hline $\boldsymbol{\Delta}$ SAIFI, \% & 9,1 & 16,0 & - \\
\hline
\end{tabular}

\section{Conclusions}

Dependence of the economic efficiency of network companies and uninterrupted power supply is determined by the current procedure for determination of the electricity transmission services tariffs in Russia. However, when making decisions at the stage of regional power supply systems and $0.4-10 \mathrm{kV}$ distribution networks development management, these indicators are not taken into account.

To increase validity of design solutions for $10 \mathrm{kV}$ and lower distribution network, efforts were made to develop a methodology allowing to calculate the forecasted SAIDI and SAIFI indexes values when designing the distribution network development, which can complement the current methodology developed by the Ministry of Energy of Russia.

It is proposed to apply generalized indexes of the distribution network elements technical condition and relationship with the perspective uninterrupted power supply indicative indexes. The methodology was tested on the example of the distribution network section. By comparing the results with the calculations performed using ETAP programming and computing suite, reliability of the obtained results was proved: deviations did not exceed $5 \%$.

Availability of the forecasted SAIDI and SAIFI indexes values in project activities will contribute to increasing validity of the made decisions, including decisions on justification circuits of distributed generation during technological connection to networks, measures for the creation of local power systems and their interconnection taking into account equipment technical condition indexes, structural and functional reliability of the distribution network and the existing operating restrictions, which is a necessary element of development management at the stage of design.

This work was supported by the Novosibirsk State Technical University (Project C20-16).

\section{References}

1. Electrical Safety Rules. Edition 6 (Moscow, 2016)

2. Government of the Russian Federation (31.12.2009),

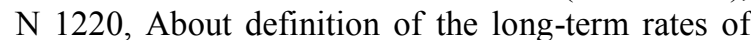
reliability indicators and quality of the delivered 
goods and the rendered services applied at establishment

3. Ministry of Energy of Russian Federation (26.07. 2017), N 676, Methodology for assessing technical condition of the main technological equipment and power transmission lines of power plants and electric networks

4. Ministry of Energy of Russian Federation (19.02.2019), N 123, Methodological guidelines for calculating the probability of failure of a functional unit and a piece of the main technological equipment and assessing consequences of such a failure

5. F. L. Byk, L. S. Myshkina, I, A, Sabadash IOP Conf. Ser.: Mater. Sci. Eng. 791, 01204 (2020)

6. T. Saati Making decisions. Method of hierarchy analysis, Radio and communication (1993)

7. A. Nazarychev, A. Tadzhibaev, D. Andreyev E3S Web of Conferences, 25 (2017)

8. Rosseti Siberia is preparing a platform for the transition to network management digital model [Online] Available: https://rosseti-sib.ru (Accessed on: May 15, 2020)

9. A.Khokhlov, Y.Melnikov, F.Veselov, D.Kholkin, K. Datsko Distributed energy in Russia: Development Potential [Online] Available: http://www.energosovet.ru/stat/skolkovo_914.pdf (Accessed on: May 13, 2020)

10. F. L. Byk, L. Myshkina, K. N. Khokhlova Advances in Engineering Research Actual "Issues of Mechanical Engineering", 133, 525-530 (2017)

11. A.G.Fishov, Yu.V. Kakosha The International Scientific and Practical Conference: Alternative and Intellectual Energy, 2, 18-20 (Voronezh, 2018).

12. A.G.Fishov, N.N.Lizalek, Y.V.Kakosha, I.L. Klavsuts D. A.Klavsuts International universities power engineering conference UPEC, 54 (Bucharest, 2019) 